\title{
ON THE BEHAVIOUR OF THE GHARAGTERISTIC FUNCTION OF A PROBABILITY DISTRIBUTION IN THE NEIGHBOURHOOD OF THE ORIGIN
}

\author{
E. J. G. PITMAN 1
}

(Received 20 April 1967)

\section{Introduction}

Let $X$ be a real valued random variable with probability measure $P$ and distribution function $F$. It will be convenient to take $F$ as the intermediate distribution function defined by

$$
F(x)=\frac{1}{2}[P\{X<x\}+P\{X \leqq x\}] .
$$

In mathematical analysis it is a little more convenient to use this function rather than

$$
F_{1}(x)=P\{X<x\} \text { or } F_{2}(x)=P\{X \leqq x\},
$$

which arise more naturally in probability theory. In all cases we shall consider

$$
\begin{aligned}
F_{1}(x) & \sim F_{2}(x) \sim F(x), & & x \rightarrow-\infty, \\
1-F_{1}(x) & \sim 1-F_{2}(x) \sim 1-F(x), & & x \rightarrow \infty .
\end{aligned}
$$

With this definition, if the distribution function of $X$ is $F(x)$, then the distribution function of $-X$ is $1-F(-x)$. The distribution of $X$ is symmetrical about 0 if $F(x)=1-F(-x)$.

The characteristic function of $X$, or of $F$, is $\phi$, defined for all real $t$ by

$$
\phi(t)=\int_{-\infty}^{\infty} e^{i t x} d F(x) .
$$

This paper deals with the relation between the value of $F(x)$ for large $x$ and the value of $\phi(t)$ for small $t$. We are interested in the behaviour of $\phi(t)$ in the neighbourhood of $t=0$ because upon this depend all limit theorems on sums of random variables. Most of the theorems proved here were stated without proofs in [1].

For $x \geqq 0$, put

1 This research was supported in part by the Office of Naval Research Contract No. Nonr 4010(09). 


$$
\begin{aligned}
& H(x)=1-F(x)+F(-x), \quad \text { the tail sum, } \\
& K(x)=1-F(x)-F(-x), \quad \text { the tail difference. }
\end{aligned}
$$

If the distribution is symmetrical about 0 , then $K(x)$ is identically zero. If $X$ is a non-negative random variable, $F(x)=0$ when $x<0$, and $K(x)=H(x)$ for $x>0$.

We may write

$$
\phi(t)=\int_{-\infty}^{0} e^{i t x} d F(x)+\int_{0}^{\infty} e^{i t x} d[F(x)-1] .
$$

Integrating by parts, and putting

where

$$
\phi(t)=U(t)+i V(t)
$$

$$
U(t)=\int_{-\infty}^{\infty} \cos t x d F(x), \quad V(t)=\int_{-\infty}^{\infty} \sin t x d F(x),
$$

we finally obtain

$$
\begin{aligned}
\frac{1-U(t)}{t} & =\int_{0}^{\infty} H(x) \sin t x d x, \\
\frac{V(t)}{t} & =\int_{0}^{\infty} K(x) \cos t x d x .
\end{aligned}
$$

We have the inversion formulae,

$$
\begin{aligned}
& H(x)=\frac{2}{\pi} \int_{0}^{\infty} \frac{1-U(t)}{t} \sin x t d t, \\
& K(x)=\frac{2}{\pi} \int_{0}^{\infty} \frac{V(t)}{t} \cos x t d t .
\end{aligned}
$$

$U(t)$ depends only on $H$, and $V(t)$ depends only on $K$, and $H$ and $K$ are not closely connected. The only connections between $H$ and $K$ are the relations, $H(x) \geqq|K(x)|$, and $H(x) \pm K(x)$ both non-increasing functions of $x$. In investigating the behaviour of $\phi(t)$ in the neighbourhood of $t=0$, it is therefore advisable to consider $U(t)$ and $V(t)$ separately.

Consider $H(x)$ and $U(t)$. The sort of result we are interested in is

$$
1-U(t) \sim c H(1 / t),
$$

where $c$ is a constant depending on the distribution. If the distribution has finite second moment $\mu_{2}$, then

Hence

$$
U(t)=1-\frac{1}{2} \mu_{2} t^{2}+o\left(t^{2}\right), \quad t \rightarrow 0 .
$$

$$
1-U(t) \sim \frac{1}{2} \mu_{2} t^{2},
$$$$
t \rightarrow 0
$$ 
In order to get a result of the type of (1) we must have a distribution of infinite variance.

\section{Functions of regular growth}

It is shown in [1] that we can expect a result of type (1) only when the tail sum $H(x)$ has the property that for every $\lambda>0$,

$$
\frac{H(\lambda x)}{H(x)} \rightarrow \lambda^{k}
$$

as $x \rightarrow \infty$.

We shall express this property of $H$ by saying that $H(x)$ is of index $k$ as $x \rightarrow \infty$. Functions with this property were studied by Karamata [2], [3], who showed that if $G(x)$ is integrable over any finite interval with lower endpoint $b$, a necessary and sufficient condition for $G(x)$ to be of index $k,>-1$, as $x \rightarrow \infty$ is

$$
\frac{\int_{b}^{x} G(u) d u}{x G(x)} \rightarrow \frac{1}{k+1}
$$$$
\text { as } x \rightarrow \infty \text {. }
$$

He also showed, what can easily be deduced from (2), that if $G(x)$ is of index $k, \neq 0$, as $x \rightarrow \infty$, then

$$
G(x) \sim G_{1}(x),
$$$$
x \rightarrow \infty,
$$

where $G_{1}(x)$ is a monotonic function of $x$, clearly non-decreasing if $k$ is positive, and non-increasing if $k$ is negative.

A function $L(x)$ of index 0 is sometimes called a function of slow growth. It has the property that $L(\lambda x) / L(x) \rightarrow 1$ as $x \rightarrow \infty$ for every $\lambda>0$. The functions $\log x, \log \log x$ are of index 0 , and so is any function with a finite non-zero limit as $x \rightarrow \infty$. Clearly, if $G(x)$ is of index $k$, then $G(x) / x^{k}$ is of index 0 , and so

where $L(x)$ is of index 0 .

$$
G(x)=x^{k} L(x)
$$

We say that a function $G(x)$ is of index $k$ as $x \downarrow 0$ if for every $\lambda>0$,

$$
\frac{G(\lambda x)}{G(x)} \rightarrow \lambda^{k}
$$

as $x \downarrow 0$.

LEMma 1. Let $G(w)$ be positive for $w>b$, bounded in any finite positive interval, and of index $k$ when $w \rightarrow \infty$. Let $c$ be greater than 0 . If $k>0$, then $B$ exists such that

$$
\frac{G(\lambda w)}{G(w)} \quad \text { is bounded for } w \geqq B, 0<\lambda \leqq c \text {. }
$$


If $k<0$, then $B$ exists such that

$$
\frac{G(\lambda w)}{G(w)} \quad \text { is bounded for } w \geqq B, \lambda \geqq c .
$$

Proof. If $k>0$, then as stated in $(3), G(w) \sim G_{1}(w), w \rightarrow \infty$, where $G_{1}(w)$ is a non-decreasing function of $w$. We may choose $G_{1}(w)$ so that it is bounded in any finite positive interval, and so that $G_{1}(w)>1$.

If $B$ is sufficiently great, when $w \geqq B$,

$$
\frac{1}{2} G_{1}(w)<G(w)<2 G_{1}(w) .
$$

When $\lambda w \geqq B, w \geqq B, \lambda \leqq c$,

$$
\frac{G(\lambda w)}{G(w)}<\frac{2 G_{1}(\lambda w)}{\frac{1}{2} G_{1}(w)} \leqq \frac{4 G_{1}(w)}{G_{1}(w)} .
$$

The last fraction is bounded for $w$ in any finite positive interval, and $\rightarrow 4 c^{k}$ when $w \rightarrow \infty$, and so is bounded.

Let $M=\sup \{G(w) ; 0 \leqq w \leqq B\}$. When $\lambda w<B, w \geqq B, \lambda \leqq c$,

$$
\frac{G(\lambda w)}{G(w)}<\frac{M}{\frac{1}{2} G_{1}(w)} \leqq \frac{2 M}{G_{1}(B)} .
$$

If $k<0, G(w) \sim G_{1}(w), w \rightarrow \infty$, where $G_{1}(w)$ is non-increasing and bounded in any finite positive interval. If $\lambda \geqq c$, when $w$ is great, the relations (4) hold, and as before the last fraction is bounded.

Lemma 2. Let $G(w)$ be positive and bounded for $w>b$, and let $h$ and $c$ be greater than 0 . If $G(w)$ is of index $-m$ as $w \rightarrow \infty$, where $m \geqq 0$, then $A, B$ exist such that

$$
\begin{array}{ll}
\frac{G(\lambda w)}{G(w)}<\frac{A}{\lambda^{m+h}} & \text { for } w \geqq B, 0<\lambda \leqq c, \\
\frac{G(\lambda w)}{G(w)}<\frac{A}{\lambda^{m-h}} & \text { for } w \geqq B, \lambda \geqq c .
\end{array}
$$

Proof. $w^{m+h} G(w)$ is of index $h$, and so by Lemma $1, A, B$ exist such that

and therefore

$$
\frac{(\lambda w)^{m+h} G(\lambda w)}{w^{m+h} G(w)}<A \quad \text { for } w \geqq B, 0<\lambda \leqq c,
$$

$$
\frac{G(\lambda w)}{G(w)}<\frac{A}{\lambda^{m+h}} \quad \text { for } w \geqq B, 0<\lambda \leqq c .
$$

The other result is obtained similarly. 
LEMMA 3. If $G(w)$ is monotonic for $w>a$, and $\int_{a}^{w} u^{r} G(u) d u$ is of index $k$, $w \rightarrow \infty$, where $k>0$, then $w^{r} G(w)$ is of index $k-1$.

Proof. If $b>0, c>0, b \neq c$,

$$
\int_{b w}^{c w} u^{r} G(u) d u
$$

is of index $k, w \rightarrow \infty$. Take $\mu>1, \lambda>0$. Without loss of generality we may assume that $G(w)$ is positive when $w>a$. We shall give the proof for $G(w)$ non-increasing and $r \geqq 0$. The proofs for the other cases are similar.

When $\lambda w>a, w>\mu a$,

$$
\frac{\int_{\lambda w}^{\lambda \mu w} u^{r} G(u) d u}{\int_{w / \mu}^{w} u^{r} G(u) d u} \leqq \frac{\lambda \mu(\lambda \mu w)^{r} G(\lambda w)}{(w / \mu)^{r} G(w)}=\frac{\lambda^{r+1} \mu^{2 r+1} G(\lambda w)}{G(w)}
$$

When $w \rightarrow \infty$, the first expression in $(5) \rightarrow(\lambda \mu)^{k}$. Hence

$$
\liminf _{w \rightarrow \infty} \frac{G(\lambda w)}{G(w)} \geqq \frac{(\lambda \mu)^{k}}{\lambda^{r+1} \mu^{2 r+1}}=\lambda^{k-r-1} \mu^{k-2 r-1} .
$$

Making $\mu \downarrow 1$, we obtain

$$
\lim _{w \rightarrow \infty} \inf \frac{G(\lambda w)}{G(w)} \geqq \lambda^{k-r-1} .
$$

By replacing $\mu$ in the above argument by $1 / \mu$, we obtain similarly

Hence

$$
\lim _{w \rightarrow \infty} \sup _{w \rightarrow \infty} \frac{G(\lambda w)}{G(w)} \leqq \lambda^{k-r-1}
$$

$$
\lim _{w \rightarrow \infty} \frac{G(\lambda w)}{G(w)}=\lambda^{k-r-1} .
$$

Thus $G$ is of index $k-r-1$ at $\infty$, and so $w^{r} G(w)$ is of index $k-1$.

\section{Relations between $\boldsymbol{H}$ and $\boldsymbol{U}$}

It is easy to show that if the distribution has infinite second moment, and if $H(x)$ is of index $k$ as $x \rightarrow \infty$, then $-2 \leqq k \leqq 0$.

Write

$$
\begin{aligned}
S(m) & =\frac{\frac{1}{2} \pi}{\Gamma(m) \sin \frac{1}{2} m \pi}, & & m>0, \\
& =1, & & m=0, \\
C(m) & =\frac{\frac{1}{2} \pi}{\Gamma(m) \cos \frac{1}{2} m \pi}, & & m>0 .
\end{aligned}
$$


$S(m)$ is finite for $m$ not an even positive integer, and, for $0<m<2$, $^{2}$

$$
S(m)=\int_{0}^{\infty} \frac{\sin x}{x^{m}} d x .
$$

If $2 n<m<2 n+2$, where $n$ is a positive integer,

$$
S(m)=\int_{0}^{\infty}\left\{\sin x-\sum_{1}^{n}(-1)^{r-1} \frac{x^{2 r-1}}{(2 r-1) !}\right\} x^{-m} d x .
$$

$C(m)$ is finite for $m$ not an odd positive integer, and for $0<m<1$,

$$
C(m)=\int_{0}^{\infty} \frac{\cos x}{x^{m}} d x .
$$

If $2 n-1<m<2 n+1$, where $n$ is a positive integer,

$$
C(m)=\int_{0}^{\infty}\left\{\cos x-\sum_{0}^{n-1}(-1)^{r} \frac{x^{2 r}}{(2 r) !}\right\} x^{-m} d x .
$$

THEOREM 1. If $H(x)$ is of index $-m$ when $x \rightarrow \infty$, and $0<m<2$, then

$$
\mathbf{l}-U(t) \sim S(m) H(\mathbf{l} / t)
$$

Proof.

$$
\begin{aligned}
& \frac{1-U(t)}{t}=\int_{0}^{\infty} H(x) \sin t x d x \quad(t>0), \\
& \frac{\mathrm{l}-U(t)}{H(\mathrm{l} / t)}=\int_{0}^{\infty} \frac{H(x / t)}{H(\mathrm{l} / t)} \sin x d x \quad(t>0) .
\end{aligned}
$$

If $0<x \leqq p$, it follows from Lemma 2 , that if $h>0$, when $t$ is sufficiently small,

$$
\frac{H(x / t)}{H(1 / t)}|\sin x| \leqq \frac{A|\sin x|}{x^{m+h}},
$$

where $A$ is a finite constant. We can choose $h$ so that $m+h<2$. The last function is then integrable over the finite interval $(0, p)$. When $t \downarrow 0$, $H(x / t) / H(1 / t) \rightarrow x^{-m}$ for $x>0$. Therefore

$$
\int_{0}^{p} \frac{H(x / t)}{H(\mathbf{l} / t)} \sin x d x \rightarrow \int_{0}^{p} \frac{\sin x}{x^{m}} d x
$$

By the Second Mean Value Theorem

$$
\int_{p}^{\infty} \frac{H(x / t)}{H(1 / t)} \sin x d x=\frac{H(p / t)}{H(1 / t)} \int_{p}^{q} \sin x d x,
$$

2 See [4] page 260, Ex. 12 for this and for the first result below for $C(m)$. The results for higher values of $m$ are derived from those for lower values by integration by parts. 
which has a modulus $\leqq 2 H(p / t) / H(1 / t)$. This $\rightarrow 2 / p^{m}$ when $t \downarrow 0$, and so can be made arbitrarily small by making $p$ sufficiently great and $t$ sufficiently small. It follows that when $t \downarrow 0$, the integral in (6) tends to

$$
\int_{0}^{\infty} \frac{\sin x}{x^{m}} d x
$$

This proves Theorem 1 .

THEOREM 2. If $H(x)$ is of index 0 as $x \rightarrow \infty$, and

$$
H(x+h) \leqq \frac{1}{2}[H(x)+H(x+2 h)]
$$

when $x$ and $h$ are sufficiently great, then

$$
1-U(t) \sim H(\mathbf{1} / t),
$$

This is the extension of Theorem 1 to the case $m=0$. It appears from the counter-example in Section 6 at the end of this paper that some additional condition such as (7) is required for $m=0$. The condition will be satisfied if $H(x)$ is convex when $x$ is sufficiently great.

Proof. Suppose that (7) is true when $x, h \geqq B$. Let $C$ be a real number such that $H(C) \leqq \frac{1}{2} H(B)$. (7) is true when $x \geqq B, h \geqq C$. When $x<B$, $h \geqq C$,

$$
2 H(x+h) \leqq 2 H(C) \leqq H(B) \leqq H(x)+H(x+2 h) .
$$

Thus (7) is true for all $x \geqq 0$ and all $h \geqq C$.

$$
\begin{aligned}
\frac{1-U(t)}{H(\mathbf{1} / t)} & =\int_{0}^{\infty} \frac{H(x / t) \sin x}{H(1 / t)} d x=\sum_{0}^{\infty} \int_{n \pi}^{(n+1) \pi} \\
& =\int_{0}^{\pi} \frac{\sin x}{H(1 / t)}\left[\sum_{n=0}^{\infty}(-1)^{n} H\left\{\frac{x+n \pi}{t}\right\}\right] d x .
\end{aligned}
$$

If

where

$$
U=u_{1}-u_{2}+u_{3}-\cdots
$$

$$
u_{n}-u_{n+1} \geqq u_{n+1}-u_{n+2} \geqq 0, \quad(n=1,2, \cdots)
$$

and $u_{n} \rightarrow 0$ as $n \rightarrow \infty$, then

Therefore

$$
U \geqq u_{2}-u_{3}+u_{4}-\cdots=u_{1}-U
$$

Again

$$
U \geqq \frac{1}{2} u_{1} .
$$

$$
\begin{aligned}
U & =u_{1}-\left(u_{2}-u_{3}+u_{4}-\cdots\right) \\
& \leqq u_{1}-\frac{1}{2} u_{2} .
\end{aligned}
$$


Thus

$$
\frac{1}{2} u_{1} \leqq U \leqq u_{1}-\frac{1}{2} u_{2} .
$$

We have shown that when $h$ is sufficiently great

$$
2 H(x+h) \leqq H(x)+H(x+2 h),
$$

i.e.

$$
H(x)-H(x+h) \geqq H(x+h)-H(x+2 h) .
$$

Therefore, when $t$ is sufficiently small,

$$
H\left\{\frac{x+n \pi}{t}\right\}-H\left\{\frac{x+(n+1) \pi}{t}\right\} \geqq H\left\{\frac{x+(n+1) \pi}{t}\right\}-H\left\{\frac{x+(n+2) \pi}{t}\right\} .
$$

Hence when $t$ is sufficiently small,

and therefore

$$
\frac{1}{2} H(x / t) \leqq \sum_{n=0}^{\infty}(-1)^{n} H\left\{\frac{x+n \pi}{t}\right\} \leqq H(x / t)-\frac{1}{2} H\left\{\frac{x+\pi}{t}\right\},
$$

$$
\frac{1}{2} I_{1} \leqq \frac{1-U(t)}{H(1 / t)} \leqq I_{1}-\frac{1}{2} I_{2}
$$

where

$$
I_{1}=\int_{0}^{\pi} \frac{H(x / t)}{H(1 / t)} \sin x d x, \quad I_{2}=\int_{0}^{\pi} \frac{H\{(x+\pi) / t\}}{H(1 / t)} \sin x d x .
$$

Both integrands $\rightarrow \sin x$ when $t \downarrow 0$. The second integrand is dominated by $\sin x$. From Lemma 2 we see that the first integrand is dominated by $A \sin x / x^{\frac{1}{2}}$. Hence $I_{1}, I_{2}$ both tend to

$$
\int_{0}^{\pi} \sin x d x,=2 .
$$

Theorem 2 then follows from (8).

Theorem $3{ }^{3}$ If $H(x)$ is of index -2 as $x \rightarrow \infty$, then

$$
1-U(t) \sim t^{2} \int_{0}^{1 / t} x H(x) d x
$$

Proof.

$$
\frac{\mathbf{1}-U(t)}{H(\mathbf{l} / t)}=\int_{\mathbf{0}}^{\infty} \frac{H(x / t)}{H(\mathbf{l} / t)} \sin x d x .
$$

Here $H$ is of index -2 , and so for $x>0$,

$$
\frac{H(x / t)}{H(1 / t)} \rightarrow \frac{1}{x^{2}}
$$

Also, if $c>0$, and $t$ positive and sufficiently small, we see from Lemma 2 with $h=\frac{1}{2}$, that

${ }^{3}$ A stronger result than this can be obtained by putting $n=0$ in Theorem 6 (iii). 


$$
\frac{H(x / t)}{H(1 / t)} \leqq \frac{A}{x^{\frac{3}{2}}}
$$

when $x \geqq c$.

Therefore

$$
\int_{c}^{\infty} \frac{H(x / t)}{H(1 / t)} \sin x d x \rightarrow \int_{c}^{\infty} \frac{\sin x}{x^{2}} d x
$$

The last integral $\rightarrow \infty$ when $c \downarrow 0$, and therefore

$$
\int_{0}^{\infty} \frac{H(x / t)}{H(\mathbf{1} / t)} \sin x d x \rightarrow \infty
$$

Hence

and so

$$
\int_{0}^{\infty} \frac{H(x / t)}{H(1 / t)} \sin x d x \sim \int_{0}^{c} \frac{H(x / t)}{H(1 / t)} \sin x d x, \quad t \downarrow 0
$$

$$
\int_{0}^{\infty} H(x / t) \sin x d x \sim \int_{0}^{c} H(x / t) \sin x d x,
$$

Since $c$ may be arbitrarily small, this must be

Thus

$$
\sim \int_{0}^{c} H(x / t) x d x \sim \int_{0}^{1} H(x / t) x d x
$$

$1-U(t)=\int_{0}^{\infty} H(x / t) \sin x d x \sim \int_{0}^{1} H(x / t) x d x \sim t^{2} \int_{0}^{1 / t} H(x) x d x, \quad t \downarrow 0$.

THEOREM 4. Let $U_{1}(t)$ be the real part at $t$ of the characteristic function corresponding to the tail function $H_{1}$. If $H$ satisfies the conditions of Theorem 1,2 or 3 then

$$
\begin{aligned}
& H_{1}(x)=O\{H(x)\}, x \rightarrow \infty \Rightarrow 1-U_{1}(t)=O\{1-U(t)\}, t \downarrow 0, \\
& H_{1}(x)=o\{H(x)\}, x \rightarrow \infty \Rightarrow 1-U_{1}(t)=o\{1-U(t)\}, t \downarrow 0 .
\end{aligned}
$$

Proof. Consider the relation $H_{1}(x)=O\{H(x)\}$, and suppose $H_{1}(x) \leqq k H(x)$.

$$
1-U_{1}(t)=\int_{0}^{\infty} H_{1}(x / t) \sin x d x \leqq \int_{0}^{\pi} H_{1}(x / t) \sin x d x,
$$

because $H_{1}(x)$ is a non-increasing function of $x$. Hence

$$
\begin{aligned}
1-U_{1}(t) \leqq & k \int_{0}^{\pi} H(x / t) \sin x d x=k H(1 / t) \int_{0}^{\pi} \frac{H(x / t)}{H(1 / t)} \sin x d x \\
& \sim k H(1 / l) \int_{0}^{\pi} \frac{\sin x}{x^{m}} d x \sim c(1-U(t)
\end{aligned}
$$

if $m<2$, where $c$ is a constant. When $m=2$ 


$$
\begin{aligned}
1-U_{1}(t) & \leqq k \int_{0}^{\pi} H(x / t) \sin x d x=k t \int_{0}^{\pi i t} H(x) \sin t x d x \\
& \leqq k t^{2} \int_{0}^{\pi / t} H(x) x d x \sim k t^{2} \int_{0}^{1 / t} H(x) x d x \sim k\{1-U(t)\}, \quad t \downarrow 0 .
\end{aligned}
$$

This proves the first part of the theorem, and the second part is proved similarly.

Theorem 5. If $1-U(t)$ is of index $m$ as $t \downarrow 0$ and $0 \leqq m<2$, then

and if $m=2$, then

$$
H(x) \sim \frac{1-U(1 / x)}{S(m)}, \quad x \rightarrow \infty,
$$

$$
\int_{0}^{x} u H(u) d u \sim x^{2}\{1-U(1 / x)\} \quad x \rightarrow \infty .
$$

Proof. For $x \geqq 0$ define

$$
\begin{aligned}
& H_{1}(x)=\int_{0}^{x} u H(u) d u, \quad H_{2}(x)=\int_{0}^{x} H_{1}(u) d u . \\
& H(x)=\frac{2}{\pi} \int_{0}^{\infty} \frac{1-U(t)}{t} \sin x t d t \\
& H_{1}(x)=\frac{2}{\pi} \int_{0}^{x} \int_{0}^{\infty} \frac{1-U(t)}{t} u \sin u t d t d u .
\end{aligned}
$$

The integrand is bounded, and

$$
\int_{0}^{T} \frac{1-U(t)}{t} u \sin u t d t
$$

is bounded in $0 \leqq u \leqq x, T \geqq 0$. See (a) below. We may therefore reverse the order of integration, and so

$$
\begin{aligned}
H_{1}(x) & =\frac{2}{\pi} \int_{0}^{\infty} \int_{0}^{x} \frac{1-U(t)}{t} u \sin u t d u d t \\
& =\frac{2}{\pi} \int_{0}^{\infty}\{1-U(t)\} \frac{\sin x t-x t \cos x t}{t^{3}} d t .
\end{aligned}
$$

We now have an absolutely convergent integral.

$$
H_{2}(x)=\frac{2}{\pi} \int_{0}^{x} \int_{0}^{\infty}\{1-U(t)\} \frac{\sin u t-u t \cos u t}{t^{3}} d t d u .
$$

By Fubini's theorem we may reverse the order of integration, and so

$$
H_{2}(x)=\frac{2}{\pi} \int_{0}^{\infty}\{1-U(t)\} \frac{2(1-\cos x t)-x t \sin x t}{t^{4}} d t .
$$


Hence

$$
\frac{H_{2}(x)}{x^{3}\{1-U(1 / x)\}}=\frac{2}{\pi} \int_{0}^{\infty} \frac{1-U(t / x)}{1-U(1 / x)} \frac{2(1-\cos t)-t \sin t}{t^{4}} d t .
$$

$1-U(1 / x)$ is of index $-m$ as $x \rightarrow \infty$. Hence by Lemma 2 , with

$$
w=x, G(x)=1-U(1 / x), \lambda=1 / t, c=1
$$

when $x$ is sufficiently great,

$$
\begin{aligned}
\frac{1-U(t / x)}{1-U(1 / x)} & <A t^{m+h} & & \text { when } t \geqq 1, \text { and } \\
& <A t^{m-h} & & \text { when } t<1,
\end{aligned}
$$

where $h>0$. If $0 \leqq m<2$, we can choose $h$ so that $h<1, m+h<2$. The integrand will then be dominated by

$$
A\left(t^{m+h}+t^{m-h}\right) \frac{2(1-\cos t)-t \sin t}{t^{4}},
$$

which is integrable over $(0, \infty)$. When $x \rightarrow \infty$

Therefore

$$
\frac{1-U(t / x)}{1-U(1 / x)} \rightarrow t^{m}
$$

$$
\frac{H_{2}(x)}{x^{3}\{1-U(1 / x)\}} \rightarrow \frac{2}{\pi} \int_{0}^{\infty} \frac{2(1-\cos t)-t \sin t}{t^{4-m}} d t,=g(m) .
$$

Thus

$$
H_{2}(x) \sim g(m) x^{3}\{1-U(1 / x)\}, \quad x \rightarrow \infty .
$$

It can be shown that

$$
g(m)=\frac{1}{(3-m)(2-m) S(m)} ;
$$

but we do not need this evaluation to prove the theorem.

The relation (10) shows that $H_{2}(x)$ is of index $3-m$ as $x \rightarrow \infty$. Hence by Lemma $3, H_{1}(x)$ is of index $2-m$, and $x H(x)$ is of index $1-m$. Therefore $H(x)$ is of index $-m$, and the stated result will follow from Theorem 1 if $0<m<2$. We cannot argue in this way when $m=0$ because of the additional convexity condition in Theorem 2 . We may get over the difficulty by using the result obtained by Karamata in [2], that if $G(x)$ is integrable over any finite positive interval, and is of index $n, x \rightarrow \infty$, where $n>-1$, then

$$
\int_{0}^{x} G(u) d u \sim \frac{x G(x)}{n+1}, \quad x \rightarrow \infty .
$$


When $m=0, H(x)$ is of index 0 . Therefore $x H(x)$ is of index 1 , and

$$
\begin{aligned}
& H_{1}(x) \sim x^{2} H(x) / 2, \\
& H_{2}(x) \sim x H_{1}(x) / 3 \sim x^{3} H(x) / 6,
\end{aligned}
$$

and therefore it follows from (10) that

$$
H(x) \sim 6 g(0)\{1-U(1 / x)\}=1-U(1 / x) .
$$

That $6 g(0)=1$ follows from Theorem 2 or from (11).

When $m=2$, we consider $H_{3}$, defined by

$$
H_{3}(x)=\int_{0}^{x} H_{2}(u) d u \text {. }
$$

We can show that

$$
H_{3}(x) \sim k x^{4}\{1-U(1 / x)\}, \quad x \rightarrow \infty,
$$

where $k$ is a constant. Thus $H_{3}$ is of index 2 at $\infty$. Hence, by Lemma 3, $H_{2}$ is of index 1 , and $H_{1}$ of index 0 at $\infty$. Using Karamata's result (12), we obtain

and so

$$
\begin{aligned}
& H_{2}(x) \sim x H_{1}(x), \\
& x \rightarrow \infty \\
& H_{3}(x) \sim \frac{1}{2} x H_{2}(x) \sim \frac{1}{2} x^{2} H_{1}(x), \\
& x \rightarrow \infty
\end{aligned}
$$

$$
H_{1}(x) \sim 2 k x^{2}\{1-U(1 / x)\},
$$$$
x \rightarrow \infty .
$$

Theorem 3 shows that $2 k=1$, and so (9) is proved.

$$
\begin{aligned}
\int_{0}^{T} \frac{1-U(t)}{t} \sin u t d t & =\int_{0}^{T}\left\{\int_{0}^{\infty} H(y) \sin y t \sin u t d y\right\} d t \\
& =\int_{0}^{\infty}\left\{\int_{0}^{T} H(y) \sin y t \sin u t d t\right\} d y
\end{aligned}
$$

because $\left|\int_{0}^{Y} H(y) \sin y t \sin u t d y\right| \leqq 4 u$ for $Y>0$. See (b). Thus

$$
\begin{aligned}
\int_{0}^{T} \frac{1-U(t)}{t} \sin u t d t & =\int_{0}^{\infty}\left\{\int_{0}^{T} \frac{1}{2} H(y)\{\cos (y-u) t-\cos (y+u) t\} d t\right\} d y \\
& =\int_{0}^{\infty} \frac{1}{2} H(y)\left\{\frac{\sin (y-u) T}{y-u}-\frac{\sin (y+u) T}{y+u}\right\} d y,
\end{aligned}
$$

which has a modulus $\leqq 2 A$. See (c). Thus

$$
\left|\int_{0}^{T} \frac{1-U(t)}{t} u \sin u t d t\right| \leqq 2 A x \text { for } 0 \leqq u \leqq x, \quad T>0 .
$$


(b) $\int_{0}^{Y} H(y) \sin y t \sin u t d y=\sin u t H(0) \int_{0}^{\xi} \sin y t d t+\sin u t H(Y) \int_{\xi}^{Y} \sin y t d t$, where $0 \leqq \xi \leqq Y$. The modulus of this is

$$
\begin{gathered}
\leqq|\sin u t|(2 / t+2 / t) \leqq 4 u \\
\int_{a}^{b} \frac{\sin k(y+c)}{y+c} d y
\end{gathered}
$$

is bounded for all real $a, b, c, k$, because it is equal to

$$
\int_{k(a+c)}^{k(b+c)} \frac{\sin v}{v} d v
$$

This is bounded because

$$
\int_{-\infty}^{\infty} \frac{\sin v}{v} d v
$$

exists as a finite (semi-convergent) integral. Thus

$$
\begin{gathered}
\left|\int_{a}^{b} \frac{\sin k(y+c)}{y+c} d u\right| \leqq A \\
\int_{0}^{Y} H(y) \frac{\sin k(y+c)}{y+c} d y \\
=H(0) \int_{0}^{\xi} \frac{\sin k(y+c)}{y+c} d y+H(Y) \int_{\xi}^{Y} \frac{\sin k(y+c)}{y+c} d y,
\end{gathered}
$$

where $0 \leqq \xi \leqq Y$, and so has a modulus $\leqq H(0) A+H(Y) A \leqq 2 A$.

\section{Relations between $H$ and $U$ for distributions of finite variance}

If $n$ is a positive integer,

$$
\mu_{2 n}=\int_{-\infty}^{\infty} x^{2 n} d F(x)=-\int_{0}^{\infty} x^{2 n} d H(x)=\int_{0}^{\infty} 2 n x^{2 n-1} H(x) d x .
$$
Put

THEOREM 6. Let $n$ be a non-negative integer. Suppose that $\mu_{2 n}$ is finite.

$$
U(t)=\sum_{0}^{n}(-1)^{r} \frac{\mu_{2 r} t^{2 r}}{(2 r) !}-U_{2 n}(t) .
$$

Let $H$ be of index $-m$ at $\infty$.

(i) If $2 n<m<2 n+2$, then

$$
U_{2 n}(t) \sim S(m) H(1 / t)
$$


(ii) If $m=2 n$, where $n>0$,

$$
U_{2 n}(t)-(-1)^{n} t^{2 n} \int_{1 / t}^{\infty} \frac{x^{2 n-1}}{(2 n-1) !} H(x) d x \sim(-1)^{n-1} \frac{\Gamma^{\prime}(2 n)}{\Gamma(2 n)^{2}} H(1 / t), \quad t \downarrow 0 .
$$

This implies the weaker statement,

$$
U_{2 n}(t) \sim(-1)^{n} t^{2 n} \int_{1 / t}^{\infty} \frac{x^{2 n-1}}{(2 n-1) !} H(x) d x,
$$

(iii) If $m=2 n+2$

$U_{2 n}(t)-(-1)^{n} t^{2 n+2} \int_{0}^{1 / t} \frac{x^{2 n+1}}{(2 n+1) !} H(x) d x \sim(-1)^{n} \frac{\Gamma^{\prime}(2 n+2)}{\Gamma(2 n+2)^{2}} H(1 / t), \quad t \downarrow 0$, which implies

$$
U_{2 n}(t) \sim(-1)^{n} t^{2 n+2} \int_{0}^{1 / t} \frac{x^{2 n+1}}{(2 n+1) !} H(x) d x, \quad t \downarrow 0 .
$$

Proof.

where

$$
\begin{aligned}
U_{2 n}(t) & =1-U(t)-\sum_{1}^{n}(-1)^{r-1} \frac{\mu_{2 r} t^{2 r}}{(2 r) !} \\
& =t \int_{0}^{\infty} H(x)\left\{\sin t x-\sum_{1}^{n}(-1)^{r-1} \frac{x^{2 r-1} t^{2 r-1}}{(2 r-1) !}\right\} d x \\
& =\int_{0}^{\infty} H(x / t) G_{n}(x) d x,
\end{aligned}
$$

$$
G_{n}(x)=\sin x-\sum_{1}^{n}(-1)^{r-1} \frac{x^{2 r-1}}{(2 r-1) !} .
$$

Note that $(-1)^{n} G_{n}(x)$ is positive when $x$ is positive. ${ }^{4}$ Also

$$
\begin{aligned}
&(-1)^{n} G_{n}(x) \sim \frac{x^{2 n-1}}{(2 n-1) !}, \\
&(-1)^{n} G_{n}(x) \sim \frac{x^{2 n+1}}{(2 n+1) !}, \\
& \frac{U_{2 n}(t)}{H(1 / t)}=\int_{0}^{c} \frac{H(x / t)}{H(1 / t)} G_{n}(x) d x+\int_{c}^{\infty} \frac{H(x / t)}{H(1 / t)} G_{n}(x) d x,
\end{aligned}
$$

where $c>0$.

When $0<x \leqq c, h>0$, and $t$ sufficiently small,

$$
\left|\frac{H(x / t)}{H(1 / t)} G_{n}(x)\right| \leqq \frac{A\left|G_{n}(x)\right|}{x^{m+h}}
$$

4 See [5] pages 80,81 . 
by Lemma 2. If $m<2 n+2$, we can choose $h$ so that $m+h<2 n+2$. $A G_{n}(x) / x^{m+h}$ is then integrable over $[0, c]$. When $t \downarrow 0, H(x / t) / H(1 / t) \rightarrow x^{-m}$, and therefore

$$
\int_{0}^{c} \frac{H(x / t)}{H(1 / t)} G_{n}(x) d x \rightarrow \int_{0}^{c} \frac{G_{n}(x)}{x^{m}} d x .
$$

Similarly we can show that if $m>2 n$,

$$
\int_{c}^{\infty} \frac{H(x / t)}{H(1 / t)} G_{n}(x) d x \rightarrow \int_{c}^{\infty} \frac{G(x)}{x^{m}} d x
$$

Hence, when $2 n<m<2 n+2$,

$$
\lim _{t \downarrow 0} \frac{U_{2 n}(t)}{H(1 / t)}=\int_{0}^{o} \frac{G_{n}(x)}{x^{m}} d x+\int_{c}^{\infty} \frac{G_{n}(x)}{x^{m}} d x=\int_{0}^{\infty} \frac{G_{n}(x)}{x^{m}} d x=S(m) .
$$

This proves (i).

$$
\begin{aligned}
& \frac{U_{2 n}(t)-(-1)^{n} t^{2 n+2} \int_{0}^{1 / t} \frac{x^{2 n+1}}{(2 n+1) !} H(x) d x}{H(1 / t)} \\
& =\int_{0}^{\infty} \frac{H(x / t)}{H(1 / t)} G_{n}(x) d x-(-1)^{n} \int_{0}^{1} \frac{x^{2 n+1}}{(2 n+1) !} \frac{H(x / t)}{H(1 / t)} d x \\
& =\int_{0}^{1} \frac{H(x / t)}{H(1 / t)} G_{n+1}(x) d x+\int_{1}^{\infty} \frac{H(x / t)}{H(1 / t)} G_{n}(x) d x .
\end{aligned}
$$

If $m=2 n+2$, when $t \downarrow 0$, the limit of this is

$$
\int_{0}^{1} \frac{G_{n+1}(x)}{x^{2 n+2}} d x+\int_{1}^{\infty} \frac{G_{n}(x)}{x^{2 n+2}} d x
$$

The sum of the two integrals in (13) is the limit as $h \downarrow 0$ of

$$
\begin{aligned}
\int_{0}^{1} \frac{G_{n+1}(x)}{x^{2 n+2+h}} d x & +\int_{1}^{\infty} \frac{G_{n}(x)}{x^{2 n+2+h}} d x \\
& =\int_{0}^{\infty} \frac{G_{n+1}(x)}{x^{2 n+2+h}} d x+(-1)^{n} \int_{1}^{\infty} \frac{x^{2 n+1}}{(2 n+1) ! x^{2 n+2+h}} d x \\
& =S(2 n+2+h)+\frac{(-1)^{n}}{h(2 n+1) !}
\end{aligned}
$$

The limit of this, as $h \downarrow 0$, is easily shown to be

and so (iii) is proved.

$$
\frac{(-1)^{n} \Gamma^{\prime}(2 n+2)}{\Gamma(2 n+2)^{2}}
$$

We prove (ii) simply by replacing $n$ in (iii) by $n-1$. 


\section{Relations between $K$ and $V$}

If $K$ is of index $-m$ at $\infty$, where $m \geq 0, K(\lambda x) / K(x) \rightarrow \lambda^{-m}$ as $x \rightarrow \infty$, for $\lambda>0$. Hence $K(x)$ must be either always positive or always negative when $x$ is great. We shall give the proofs always for the case $K(x)>0$ when $x$ is great.

Theorem 7. Put

where

$$
K(x)=K_{1}(x)-K_{2}(x),
$$

$$
K_{1}(x)=1-F(x), \quad K_{2}(x)=F(-x) .
$$

Suppose that $K$ is of index $-m$ at $\infty$, and that, when $x$ is great, either $K(x)$ is monotonic, or $K_{2}(x)<\lambda K_{1}(x)$, where $0<\lambda<1$.

(i) If $0<m<1$, then

$$
V(t) \sim C(m) K(\mathbf{1} / t)
$$

(ii) If $m=0$, then

$$
\int_{0}^{t} \frac{V(u)}{u} d u \sim \frac{1}{2} \pi K(1 / t), \quad t \downarrow 0 .
$$

(ii) If $m=1$, then

$$
V(t)-t \int_{0}^{1 / t} K(x) d x \sim-\gamma K(1 / t)
$$

where $\gamma$ is Euler's constant.

The proof of (i) when $K(x)$ is ultimately monotonic is similar to the proof of Theorem 1 . When we assume that $K_{2}(x)<\lambda K_{1}(x)$ when $x$ is great, a slight modification is necessary. In the course of the proof we need to show that the integral

$$
\int_{v}^{\infty} \frac{K(x / t)}{K(1 / t)} \cos x d x
$$

can be made arbitrarily small by making $p$ sufficiently great and $t$ sufficiently small.

It follows from $K_{2}(x)<\lambda K_{1}(x)$ that

$$
K_{1}(x)+K_{2}(x)<\frac{(1+\lambda) K(x)}{1-\lambda} .
$$

Using the Second Mean Value Theorem, we obtain

$$
\int_{p}^{\infty} \frac{K(x / t)}{K(1 / t)} \cos x d x=\frac{K_{1}(p / t)}{K(1 / t)} \int_{p}^{q_{1}} \cos x d x-\frac{K_{2}(p / t)}{K(1 / t)} \int_{p}^{q_{2}} \cos x d x
$$


The modulus of the right hand side is not greater than

$$
\frac{2 K_{1}(p \mid t)+2 K_{2}(p / t)}{K(1 / t)},
$$

which is less than

$$
\frac{2(1+\lambda) K(p \mid t)}{(1-\lambda) K(1 / t)}
$$

when $p / t$ is great. Since $K(p / t) / K(1 / t) \rightarrow 1 / p$ as $t \rightarrow 0$, the required result follows.

We now consider the proof of (ii).

$$
\frac{V(u)}{u}=\int_{0}^{\infty} K(x) \cos u x d x .
$$

If $t_{0}>0$, the integral is uniformly convergent with respect to $u>t_{0}$.

Therefore, if $t>t_{0}$,

$$
\begin{aligned}
\int_{t_{0}}^{t} \frac{V(u)}{u} d u & =\int_{0}^{\infty} K(x) \frac{\sin t x}{x} d x-\int_{0}^{\infty} K(x) \frac{\sin t_{0} x}{x} d x \\
& =\int_{0}^{\infty} K(x / t) \frac{\sin x}{x} d x-\int_{0}^{\infty} K\left(x / t_{0}\right) \frac{\sin x}{x} d x
\end{aligned}
$$

The second integral on the right is uniformly convergent with respect to $t_{0}>0$, and therefore $\rightarrow 0$ when $t_{0} \downarrow 0$. Hence

and

$$
\int_{0}^{t} \frac{V(u)}{u} d u=\int_{0}^{\infty} K(x / t) \frac{\sin x}{x} d x
$$

$$
\frac{\int_{0}^{t} \frac{V(u)}{u} d u}{K(1 / t)}=\int_{0}^{\infty} \frac{K(x / t)}{K(1 / t)} \frac{\sin x}{x} d x .
$$

If $m=0$, when $t \downarrow 0, K(x / t) / K(1 / t) \rightarrow 1$. Using the property that when $x$ is great, either $K(x)$ is monotonic or $K_{2}(x)<\lambda K_{1}(x)$, we can as in the proof of (i), and Theorem 1 , show that when $t \downarrow 0$, the right hand side of (14) tends to

$$
\int_{0}^{\infty} \frac{\sin x}{x} d x=\frac{1}{2} \pi
$$

This proves (ii).

$$
V(t)=\int_{0}^{\infty} K(x / t) \cos x d x
$$




$$
\begin{aligned}
\frac{V(t)-t \int_{0}^{1 / t} K(x) d x}{K(1 / t)} & =\frac{V(t)-\int_{0}^{1} K(x / t) d x}{K(1 / t)} \\
& =\int_{0}^{1} \frac{K(x / t)}{K(1 / t)}(\cos x-1) d x+\int_{1}^{\infty} \frac{K(x / t)}{K(1 / t)} \cos x d x,
\end{aligned}
$$

which tends to

$$
\int_{0}^{1} \frac{\cos x-1}{x} d x+\int_{1}^{\infty} \frac{\cos x}{x} d x
$$

when $t \downarrow 0$, if $m=1$, so that $K(x / t) / K(\mathrm{I} / t) \rightarrow 1 / x$.

The sum of the two integrals in (15) is the limit, when $\theta \rightarrow 0$, of

$$
\begin{aligned}
\int_{0}^{1} \frac{\cos x-1}{x^{1+\theta}} d x+ & \int_{1}^{\infty} \frac{\cos x}{x^{1+\theta}} d x \\
& =\int_{0}^{\infty} \frac{\cos x-1}{x^{1+\theta}} d x+\int_{1}^{\infty} \frac{d x}{x^{1+\theta}}=C(1+\theta)+1 / \theta \\
& =\frac{1}{\theta}-\frac{\frac{1}{2} \pi}{\Gamma(1+\theta) \sin \frac{1}{2} \pi \theta} .
\end{aligned}
$$

This limit of this, when $\theta \rightarrow 0$, is easily shown to be

This proves (iii).

$$
\Gamma^{\prime}(1),=-\gamma \text {. }
$$

We shall write

$$
\mu_{2 n-1}^{*}=\int_{0}^{\infty}(2 n-1) x^{2 n-2} K(x) d x
$$

when this integral exists. In all the cases we shall discuss, $K(x)$ is asymptotically monotonic, and therefore, if the integral is finite, $x^{2 n-2} K(x)$ must $\rightarrow 0$ as $x \rightarrow \infty$. When this is so, integration by parts shows that

$$
\begin{aligned}
\mu_{2 n-1}^{*} & =-\int_{0}^{\infty} x^{2 n-1} d K(x)=-\lim _{T \rightarrow \infty} \int_{0}^{T} x^{2 n-1} d K(x) \\
& =\lim _{T \rightarrow \infty} \int_{-T}^{T} x^{2 n-1} d F(x) .
\end{aligned}
$$

It is possible for the integral in (16) to be finite and the limit in (17) not to exist; but not in the cases we are investigating.

It follows from Lemma 2 that if $K$ is of index $-m$ at $\infty, \mu_{2 n-1}^{*}$ is finite if $m>2 n-1$, and infinite if $m<2 n-1$.

THEOREM 8. Suppose that $\mu_{2 n-1}^{*}$ exists and is finite, where $n$ is a positive integer. Put

$$
V(t)=\sum_{x}^{n}(-1)^{r-1} \frac{\mu_{2 r-1}^{*} t^{2 r-1}}{(2 r-1) !}+V_{2 n-1}(t) .
$$


Let $K$ be of index $-m$ at $\infty$

(i) If $2 n<m<2 n+1$, then

$$
V_{2 n-1}(t) \sim C(m) K(1 / t),
$$

(ii) If $m=2 n-1$, then

$$
\begin{gathered}
V_{2 n-1}(t)-(-1)^{n} t^{2 n-1} \int_{1 / t}^{\infty} \frac{x^{2 n-2}}{(2 n-2) !} K(x) d x \\
\sim(-1)^{n-1} \frac{\Gamma^{\prime}(2 n-1)}{\Gamma(2 n-1)^{2}} K(1 / t),
\end{gathered}
$$

(iii) If $m=2 n+1$,

$$
\begin{aligned}
V_{2 n-1}(t) & -(-1)^{n} t^{2 n+1} \int_{0}^{1 / t} \frac{x^{2 n}}{(2 n) !} K(x) d x \\
& \sim(-1)^{n} \frac{\Gamma^{\prime}(2 n+1)}{\Gamma(2 n+1)^{2}} K(1 / t)
\end{aligned}
$$

The proofs are similar to those of Theorem 6 . Here

where

$$
V_{2 n-1}(t)=\int_{0}^{\infty} K(x / t) J_{n}(x) d x,
$$

$$
J_{n}(x)=\cos x-\sum_{0}^{n-1}(-1)^{r} \frac{x^{2 r}}{(2 r) !}
$$

\section{Counter-example}

In this section we give an example where $H$ is of index 0 at $\infty$ but the conclusion of Theorem 2 is not true, i.e. $1-U(t)$ is not asymptotically equal to $H(1 / t)$ as $t \downarrow 0$.

Let $\left\{T_{n}\right\}$ be an increasing sequence of integers $>1$ such that $T_{n} / T_{n-1}$ is integral. For

define

$$
2 T_{r} \leqq x \leqq 2 T_{r+1}
$$

$$
f(x)=\frac{1}{\log \left(2 m T_{r}\right)}-\frac{1}{\log \left((2 m+2) T_{r}\right)}
$$

if

$$
\begin{aligned}
x & =(2 m+1) T_{r}, \quad m=1,2, \cdots, T_{r+1} / T_{r}-1, \\
& =0 \text { otherwise. }
\end{aligned}
$$

For

define

$$
0 \leqq x \leqq 2 T_{1}
$$




$$
f(x)=1-\frac{1}{\log \left(2 T_{1}\right)},
$$

if

$$
\begin{aligned}
x & =T_{1}, \\
& =0 \text { otherwise. }
\end{aligned}
$$

Now define

$$
H(x)=\frac{1}{2} f(x)+\sum_{y>x} f(y) .
$$

Clearly $H$ is a tail-sum function. We easily obtain

$$
H\left(2 m T_{r}\right)=\frac{1}{\log \left(2 m T_{r}\right)}, \quad m=1,2, \cdots, T_{r+1} / T_{r} .
$$

If

$$
2 m T_{r} \leqq x \leqq 2(m+1) T_{r},
$$

where $m$ is a positive integer, then

and so

$$
\frac{1}{2} x \leqq 2 m T_{r} \leqq x \leqq 2(m+1) T_{r} \leqq 2 x,
$$

$$
\frac{1}{\log \left(\frac{1}{2} x\right)} \geqq H\left(2 m T_{r}\right) \geqq H(x) \geqq H\left(2(m+1) T_{r}\right) \geqq \frac{1}{\log (2 x)} .
$$

Thus for all $x>2 T_{1}$,

$$
\frac{1}{\log \left(\frac{1}{2} x\right)} \geqq H(x) \geqq \frac{1}{\log (2 x)} .
$$

It follows that $H(x) \sim 1 / \log x, x \rightarrow \infty$. Thus $H$ is of index 0 at $\infty$

$$
\begin{aligned}
1-U\left(\pi / T_{r}\right) & =-\int_{0}^{\infty}\left(1-\cos \left(\pi x / T_{r}\right)\right) d H(x) \\
& \geqq-\int_{2 T_{r}}^{2 T_{r+1}}\left(1-\cos \left(\pi x / T_{r}\right)\right) d H(x)=-\int_{2 T_{r}}^{2 T_{r+1}} 2 d H(x) .
\end{aligned}
$$

The last equality is true because at a point of increase of $H$ in the interval $\left[2 T_{r}, 2 T_{r+1}\right], \cos \left(\pi x / T_{r}\right)=-1$. Hence

$$
1-U\left(\pi / T_{r}\right) \geqq 2\left[H\left(2 T_{r}\right)-H\left(2 T_{r+1}\right)\right] \text {. }
$$

Now $H\left(T_{r}\right) \sim 1 / \log T_{r}$, and so if we choose the sequence $\left\{T_{n}\right\}$ so that $\log T_{r+1} / \log T_{r} \rightarrow \infty$ as $r \rightarrow \infty$, the right side of (18) will be asymptotically equal to

$$
2 H\left(2 T_{r}\right) \sim 2 H\left(T_{r} / \pi\right), \quad r \rightarrow \infty .
$$

Hence $1-U\left(\pi / T_{r}\right)$ will not be asymptotically equal to $H\left(T_{r} / \pi\right)$ as $r \rightarrow \infty$, and so $1-U(t)$ will not be asymptotically equal to $H(1 / t)$ as $t \downarrow 0$.

I have to thank Professor B. C. Rennie for devising this counter example. 


\section{References}

[1] E. J. G. Pitman, 'Some theorems on characteristic functions of probability distributions'. Proc. Fourth Berkeley Symp. Math. Statist. Prob. (1960) 2, 393-402

[2] M. J. Karamata, 'Sur un mode de croissance régulière des fonctions'. Mathematica (Cluj), V. iv, (1930) $38-53$.

[3] M. J. Karamata, 'Sur un mode de croissance régulière.Théorèmes fondamentaux'. Bull. de la soc. math. de France. 61 (1933) 55-62.

[4] E. T. Whittaker and G. N. Watson, A Course of Modern Analysis (Cambridge University Press, 4th ed. 1927).

[5] C. V. Durell and A. Robson, Advanced Trigonometry (G. Bell and Sons, 1930).

The Johns Hopkins University

Baltimore, Md.

and

The University of Melbourne 